\title{
Galactic cosmic ray modulation at Mars and beyond measured with EDACs on Mars Express and Rosetta
} \author{
J. Godfrey ${ }^{5}$, and A. Johnstone ${ }^{5}$ \\ ${ }^{1}$ LATMOS/IPSL, UVSQ Université Paris-Saclay, Sorbonne Université, CNRS, Guyancourt, France \\ e-mail: elise-wright.knutsen@latmos.ipsl.fr \\ 2 European Space Agency, ESTEC, Noordwijk, The Netherlands \\ 3 School of Physics and Astronomy, University of Leicester, Leicester, UK \\ ${ }^{4}$ Institute of Experimental and Applied Physics, Christian-Albrechts-University, Kiel, Germany \\ ${ }^{5}$ European Space Agency, ESOC, Darmstadt, Germany
}

E. W. Knutsen ${ }^{1,2} \odot$, O. Witasse ${ }^{2}$, B. Sanchez-Cano ${ }^{3}$, M. Lester ${ }^{3}$, R. F. Wimmer-Schweingruber ${ }^{4}$, M. Denis ${ }^{5}$,

Received 9 March 2021 / Accepted 8 May 2021

\begin{abstract}
Galactic cosmic rays (GCRs) are an intrinsic part of the heliospheric radiation environment and an inevitable challenge to longterm space exploration. Here we show solar-cycle-induced GCR modulation at Mars in the period 2005-2020, along with GCR radial gradients, by comparing Mars Express and Rosetta engineering parameters to sunspot number time series. The engineering parameters used are the error detection and correction (EDAC) counters, cumulative counters that are triggered by charged energetic particles that cause memory errors in onboard computers. EDAC data provide a new way of gaining insight into the field of particle transport in the heliosphere; these data also allow us to complement dedicated radiation instrumentation as EDAC software is present on all spacecraft. This dataset was used to capture variations in GCRs in both space and time, yielding the same qualitative information as ground-based neutron monitors. Our analysis of the Mars Express EDAC parameter reveals a strong solar cycle GCR modulation, with a time lag of $\sim 5.5$ months. By combining Mars Express with Rosetta data, we calculate a $4.7 \pm 0.8 \%$ increase in EDAC count rates per astronomical unit, which we attribute to a radial gradient in GCR fluxes in accordance with established literature. The potential of engineering data for scientific purposes remains mostly unexplored. The results obtained from this work demonstrate, for the first time for heliophysics purposes, the usefulness of the EDAC engineering parameter, the usefulness of data mining, and the utility of keeping missions operational for many years, all of which provide complimentary data to nominal science instruments.
\end{abstract}

Key words. cosmic rays - Sun: heliosphere - interplanetary medium - methods: data analysis - space vehicles: instruments radiation: dynamics

\section{Introduction}

The heliosphere is constantly permeated by charged particles originating from outside our Solar System. The cosmic rays are of Galactic origin and are isotropically incident on the heliopause. Galactic cosmic rays (GCRs), and the related radiation exposure, are seen as some of the main challenges for future long-duration exploration, especially beyond low Earth orbit. The steady flux of energetic particles exposes spacecraft hardware and humans to radiation, the magnitude of which is heavily influenced by several factors: the solar cycle, the presence of an atmosphere, magnetic fields, and relevant spacecraft shielding (Feynman \& Gabriel 2000; Schwadron et al. 2014). Investigations of GCR variations over the course of a solar cycle and at multiple locations in the heliosphere provide valuable data for mission planning and for the improvement of our understanding of GCR variations, particle transport, and structures throughout the heliosphere.

Three factors influence the modulation of GCR intensity within the ecliptic plane: time, heliocentric distance, and heliomagnetic longitude. This last factor is outside the scope of this study as longitudinal effects are considered to be small compared to temporal and radial variations (De Simone et al. 2011). As the Sun undergoes its 11-year sunspot cycle and 22-year magnetic polarity cycle, the interplanetary magnetic field (IMF), the sunspot number (SSN), and solar wind parameters vary (Richardson et al. 2001), especially if considering time periods that are long relative to a solar rotation period. As a result, GCRs in the heliosphere are modulated in their inward diffusion by the convection of the expanding solar wind and the particle drift on the IMF, giving rise to a changing GCR flux (Gazis 1996; Modzelewska et al. 2019). Only higher-energy particles are able to penetrate into the heliosphere, causing a depressed GCR intensity within the heliosphere compared to beyond it (Wibberenz 2002). Particles with energies below a few $100 \mathrm{MeV} \mathrm{nuc}^{-1}$ are more strongly affected by solar modulation compared to particles with higher energies (Potgieter 2013). The relationship has been observed at and around the Earth since the 1950s (Alania et al. 2014). A network of ground-based neutron monitors (NMs) is used to study the temporal modulation of cosmic rays by measuring the flux of cascading particles produced by cosmic rays colliding with atmospheric atoms (Engel et al. 1992; Usoskin et al. 2017). In addition to exhibiting a temporal anti-correlation with solar activity, the GCR intensity also varies with heliocentric distance, increasing with $3-4 \%$ per AU (De Simone et al. 2011; Honig et al. 2019; Modzelewska et al. 2019; Vos \& Potgieter 2016). This gradient is highly convolved with the solar and magnetic conditions at any given time and is 
generally stronger for negative polarity periods $(A<0)$, when the magnetic field has a radial inward component in the northern hemisphere and the particle drifts are inwards along the current sheet (Burger et al. 2008), and weaker for times with positive polarity (Roussos et al. 2020).

Error detection and correction (EDAC) is a technique used on most spacecraft (Shirvani et al. 2000) to detect and correct errors in memory contents. On spacecraft, this corruption is principally due to bit flips caused by single event upsets (SEUs). An SEU can happen when an energetic particle hits a physical memory cell. If the particle deposits charge in it, a memory error can occur, which in turn can corrupt the data stored on the chips if not corrected. Such errors are caught and corrected by the EDAC algorithm. Once a correction is done, the relevant EDAC counter goes up by 1 (Shirvani et al. 2000).

The relatively steady cumulative EDAC increase is attributed to the continuous presence of cosmic rays as only highly energetic particles are expected to trigger an SEU and a subsequent count increment with the EDAC counter. Normal to elevated solar wind conditions are not expected to have any direct effects on the EDAC counter as such particle energies are much too low. However, solar energetic particle (SEP) events are known to cause irregularities such as jumps in the otherwise relatively monotone counter (see panel A in Fig. 1) and have resulted in various spacecraft failures or the forced initiation of safety modes (Limes et al. 2015). Some previous studies have explored using EDACs for the purpose of monitoring space weather, such as SEP events, with promising results (e.g., Jiggens et al. 2019). Otherwise, the EDAC parameter has been of little interest for scientific purposes thus far.

Understanding the radiation environment within our Solar System is not only key to many fundamental questions about particle transport processes within the heliosphere, it is also imperative when preparing for human and robotic space exploration, especially for human space flight beyond low Earth orbit. The extremely high energies of GCRs and the strict mass constraints in space flight represent a serious challenge (Durante \& Cucinotta 2011). Tradeoffs between added weight and shielding can be further optimised if predictive models of GCR variations are improved. At present, dedicated instrumentation on specific spacecraft is used to this end in conjunction with sophisticated modelling efforts. Nevertheless, measurements are sparse, gaps in time coverage are frequent, and the spatial distribution of measurement points is very limited. By utilising EDAC counters in addition to scientific instruments, both spatial and temporal coverage can be greatly improved. EDACs are implemented on all spacecraft, past and present, and the datasets are increasingly becoming more easily available. By combining EDAC counters with tailored scientific payloads, the radiation monitoring within our Solar System can be greatly improved. This study endeavours to showcase the utility of the EDAC counter for long-term observations of GCR variations in our heliosphere, as well as to highlight some remaining challenges. Section 2 describes the datasets used and the data treatment process, Sect. 3 presents the results, and Sect. 4 reflects on the findings before a conclusion is given in Sect. 5 .

\section{Datasets}

On June 2, 2003, Mars Express (MEX) was launched and reached Mars orbit in December of the same year (Chicarro et al. 2004). After an initial commissioning phase, nominal science operations began in mid-January 2004. As of December 2020, MEX was still operational and is expected to remain so until at least the end of 2022. The EDAC counter related to the data management system processor on MEX was utilised for this work (parameter ID NDMW0D0G), which reports increments multiple times per day. Rosetta was launched on March 2, 2004, and started its ten-year journey to reach Comet 67P/ChuryumovGerasimenko (Glassmeier et al. 2007). The spacecraft was operational and in contact with the ground from launch until June 2011, at which point Rosetta entered a hibernation period that lasted until January 2014. Subsequently, Rosetta reached its target comet in August 2014 and remained operational until September 30, 2016. Out of the 18 available EDACs on Rosetta, the attitude and orbit control system counter (parameter ID NACW0D0A) was chosen for this study because of its continuous temporal coverage (except during hibernation) and its measurement frequency, which was higher and more even than the others.

For the part of this study that involves the solar cycle modulation of GCRs at Mars, the MEX EDAC housekeeping parameter was compared with SSNs for the time period January 1, 2005 - September 17, 2020. The utilised sunspot data were downloaded from the World Data Center SILSO, Royal Observatory of Belgium, Brussels (Center 2020). For the study of GCR variations with heliocentric distance, the EDACs on MEX and Rosetta from January 1, 2005, until the end of the Rosetta mission were utilised. These EDAC parameters are archived in mission databases at the European Space Operations Centre (ESOC) for all ESA spacecraft but are not necessarily publicly available as they are not scientific datasets. For some missions (such as Rosetta and Cluster), these datasets are also available in the online public archives of the European Space Astronomy Centre (ESAC).

To validate how well the EDAC parameter is suited for GCR monitoring, the counter was also compared to ground-based NM data. Neutron monitor data from several ground stations were collected from the Bartol Research Institute's website ${ }^{1}$. The NM provides proxy measurements of GCRs by measuring secondary particles created by their interaction with the atmosphere and inside the NMs (Simpson 2000).

\section{Data processing}

The EDAC parameter is a cumulative counter. However, on a few occasions over the course of the MEX and Rosetta missions, the counter was reset to zero. For the purposes of this study, these resets were corrected for by adding the count value immediately before the reset to all subsequent data points. The measuring frequency of the EDACs is quite irregular, varying from data points every $30 \mathrm{~s}$ to times with fewer than one measurement per day on average. In order to compare MEX and Rosetta EDACs, the cumulative counter was resampled with a daily frequency, and missing days were filled by linear interpolation. For each EDAC counter, a daily EDAC count rate was calculated by finding the difference in the counter every two weeks and dividing this number by 14 days. Different time bins were tested, and a 14-day average, approximately half a solar rotation period, was found to be the best compromise for removing daily variations and highlighting the solar cycle modulation. By calculating the 14-day daily average, much of the random background fluctuations even out; nevertheless, the rate is still susceptible to short-term variation and extreme space weather events.

Daily variations are caused by random fluctuations in the GCR background, which are largely removed by the previously

http://neutronm. bartol . udel . edu 

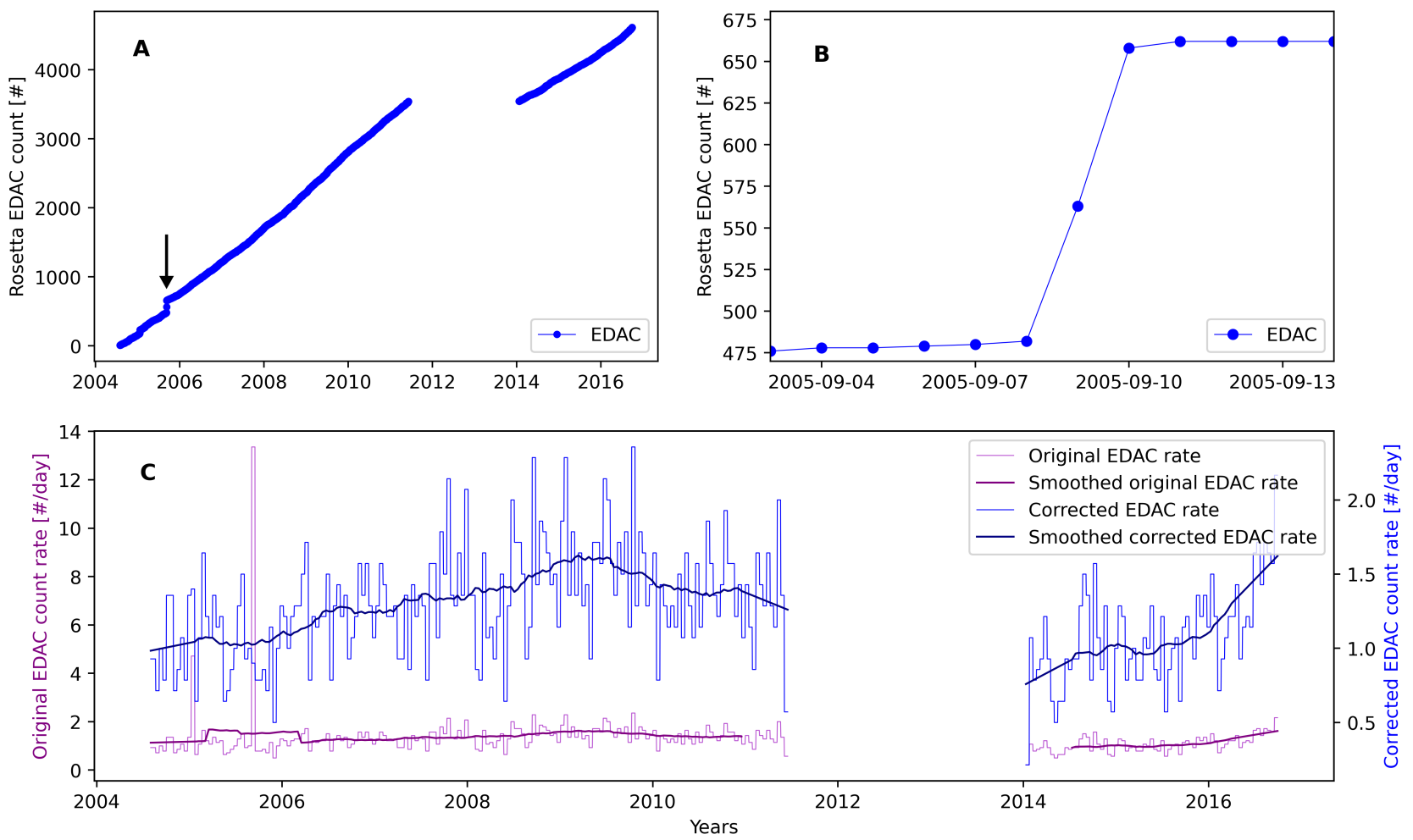

Fig. 1. Illustration of the removal process of SEP-induced disturbances (SPEs drastically influence the EDAC counters, artificially raising the calculated EDAC count rate for several months prior to and after the actual event). Panel A: Rosetta EDAC counter from January 2005 to end of mission; the blank space indicates the hibernation period and the black arrow points to an example SEP event. Panel B: close-up of the jump indicated in panel A. Panel C: smoothed and original EDAC count rates before (purple, left $y$-axis) and after (blue, right $y$-axis) removal of two distinct SEP events. We note that the original and modified count rates are plotted on different $y$-scales to highlight the extreme count rate values obtained if SEP events are not removed from the time series.

described steps, but also by the passing of energetic solar eruptions, such as coronal mass ejections or solar particle events (SPEs), that disturb the regular background of GCRs and cause irregularities in the EDAC counter. Many SPEs are detectable within these datasets; however, for both spacecraft two specific solar eruptions caused such extreme responses in the EDAC counter that it was deemed necessary to do a correction. Figure 1 illustrates this process using Rosetta as an example. In Fig. 1A the complete EDAC dataset from Rosetta is illustrated, including one clearly visible jump caused by an SPE and one smaller jump about 6 months earlier. The largest event is indicated by an arrow. This event is zoomed in on in Fig. 1B, where for two days the EDAC counter increases dramatically, with nearly 100 counts per day, compared to the 1.3 daily average. If no correction is made, the estimated 14-day moving average EDAC count rate around the point in time of the SPE leads to extreme values, as can be seen in the uncorrected EDAC count rates (purple) in Fig. 1C compared to the corrected count rates (blue). However, by replacing the EDAC rate in the 14-day interval around the SPEs with the mean of the rate before and after the event, a more realistic estimate of the GCR background can be obtained. The daily count rate average for the complete dataset before and after the extreme event correction remained very similar (reduced from $1.3 \pm 0.88$ to $1.25 \pm 0.37$ counts per day for Rosetta and from $1.39 \pm 0.48$ to $1.38 \pm 0.32$ for MEX) and is close to equal for both spacecraft.

To obtain daily count rates that reflect the long-term variations in GCR background radiation, a smoothing routine was applied after the removal of the two SEP anomalies (which occurred in January and September of 2005). A linear
Savitzky-Golay low-pass filter with a 365-day window (Savitzky \& Golay 1964) was applied to the EDAC count rate time series as well as to the SSN and NM data. In essence, this process smooths the data by removing high-frequency variations in the signal. If the SEP events had not been removed, the EDAC rates during these times would be an order of magnitude larger than the rest and the smoothed rate would be elevated to an artificially high level in the \pm 6 months surrounding the EDAC jumps, as illustrated in Fig. 1C. This would result in the solar cycle modulation of the cosmic rays appearing negligible. For studies on long timescales, this SEP-induced EDAC response removal is necessary to extract solar-induced modulation from the more intense but short term space weather phenomena. EDAC reactions to SEPs are, however, interesting in themselves. If one wanted to study these transient events, a smoothing routine with a much shorter time span would be applied. Such a study could be of use for the detection and diagnosing of space weather events, and Jiggens et al. (2019) demonstrated the feasibility of this method. Future work will investigate these responses.

\section{Results}

The datasets discussed here are EDAC counters with daily frequencies in the period from January 1, 2005, to September 17, 2020, for MEX and from January 1, 2005, to September 29, 2016, for Rosetta. Daily SSNs were compared to the MEX EDAC counter for the full duration of the MEX EDAC time period. To demonstrate the capabilities of the EDAC counter as a proxy for GCR variations, Sect. 3.1 compares the EDAC counter 

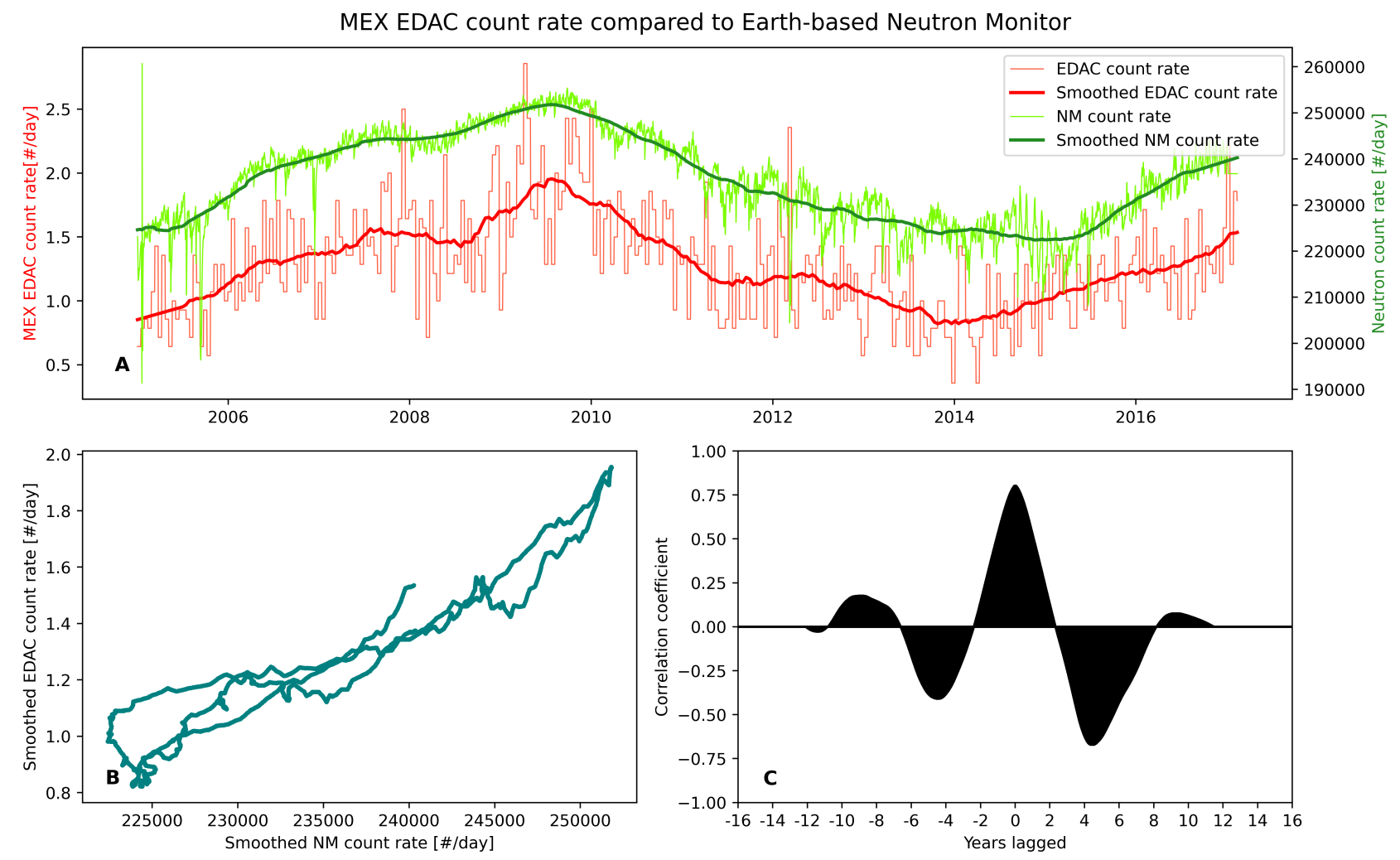

Fig. 2. Demonstration of the similar properties of the space-borne EDAC counter and the ground-based NM. Panel A: time series of the MEX EDAC count rate (red) and the McMurdo NM count rate (green). Panel B: smoothed EDAC rate as a function of the smoothed NM count rate. Panel C: cross-correlation between the two count rates; maximum correlation was found at exactly 0 lag, with a coefficient of 0.8 .

with a ground-based NM, another well-established proxy indicator of GCR activity (Usoskin et al. 1998). Section 3.2 focuses on the solar cycle modulation on cosmic rays by comparing SSNs to the MEX EDAC. The Rosetta EDAC is not used for this purpose for two reasons. First, there is an interruption in the data series during the time Rosetta was in hibernation. Second, the orbit of Rosetta took the spacecraft to large heliocentric distances, which makes any direct comparison to Earth questionable. In Sect. 3.3, however, we derive a heliocentric GCR gradient by comparing the EDAC on Rosetta with the one on board MEX.

\subsection{EDAC comparison with neutron monitor data}

All NM data used in this work were collected from the Bartol research institute, which operates eight NMs; our primary source was measurements from the McMurdo station. Data from Thule and other NM stations were also compared with the EDAC rates (not shown) and yielded nearly identical results. Figure $2 \mathrm{~A}$ directly compares the calculated MEX EDAC count rates to NM count rates, both raw and smoothed, and demonstrates that the EDAC count rate at Mars exhibits the same long-term temporal behaviour as the count rates registered at the McMurdo station on Earth. Figure 2B qualitatively confirms the linear relationship between the two count rates by plotting the smoothed EDAC count rates as a function of the smoothed NM count rates. To quantify the strength of the correlation between the two counters, a cross-correlation calculation was made, as seen in Fig. 2C. Maximum similarity between the two signals, with a coefficient of 0.8 , is obtained at a lag of 0 , indicating that if there is a delayed response of GCR modulation at Earth compared to Mars, it is undetectable with this method.
The solar cycle modulation is clearly visible in both the NM time series and the EDAC count rate, demonstrating the ability of the EDAC counter to measure the varying GCR intensity. The comparison between the two smoothed time series shows a strong correlation. Neutron monitor data is a well-established proxy for GCR measurements and makes for a good control set to validate the fitness of EDACs for the purpose of GCR variation studies. However, NMs are Earth-based instruments and are therefore influenced by magnetospheric effects - effectively being shielded from particles of lower energies to which the EDAC is still susceptible - which might cause the higher variability in the EDAC compared to the NM. This could be in part why the correlation coefficient is not even higher.

\subsection{Solar cycle modulated GCRs at Mars}

Measurements of the radiation environment around planetary bodies other than Earth are relatively sparse. The radiation field at Martian orbit was first measured in 2002 with instruments on Mars Odyssey (Zeitlin et al. 2010) and later with ExoMars Trace Gas Orbiter (Semkova et al. 2018), while measurements on the surface of Mars have been acquired since August 2012 (Hassler et al. 2012, 2014). Here we present cosmic ray variations due to solar cycle modulation in Martian orbit, for the first time recorded by engineering parameters. Figure 3 shows daily EDAC count rates and SSNs along with the smoothed time series of each dataset. All smoothing was done with a Savitzky-Golay low-pass filter, with a 365-day window and linear polynomial fit of the data. Figures $3 \mathrm{~A}$ and $\mathrm{B}$ illustrate the changing rate of EDAC increments at Mars and Rosetta, respectively, as the GCR intensity varies with the solar cycle; the SSNs are shown 

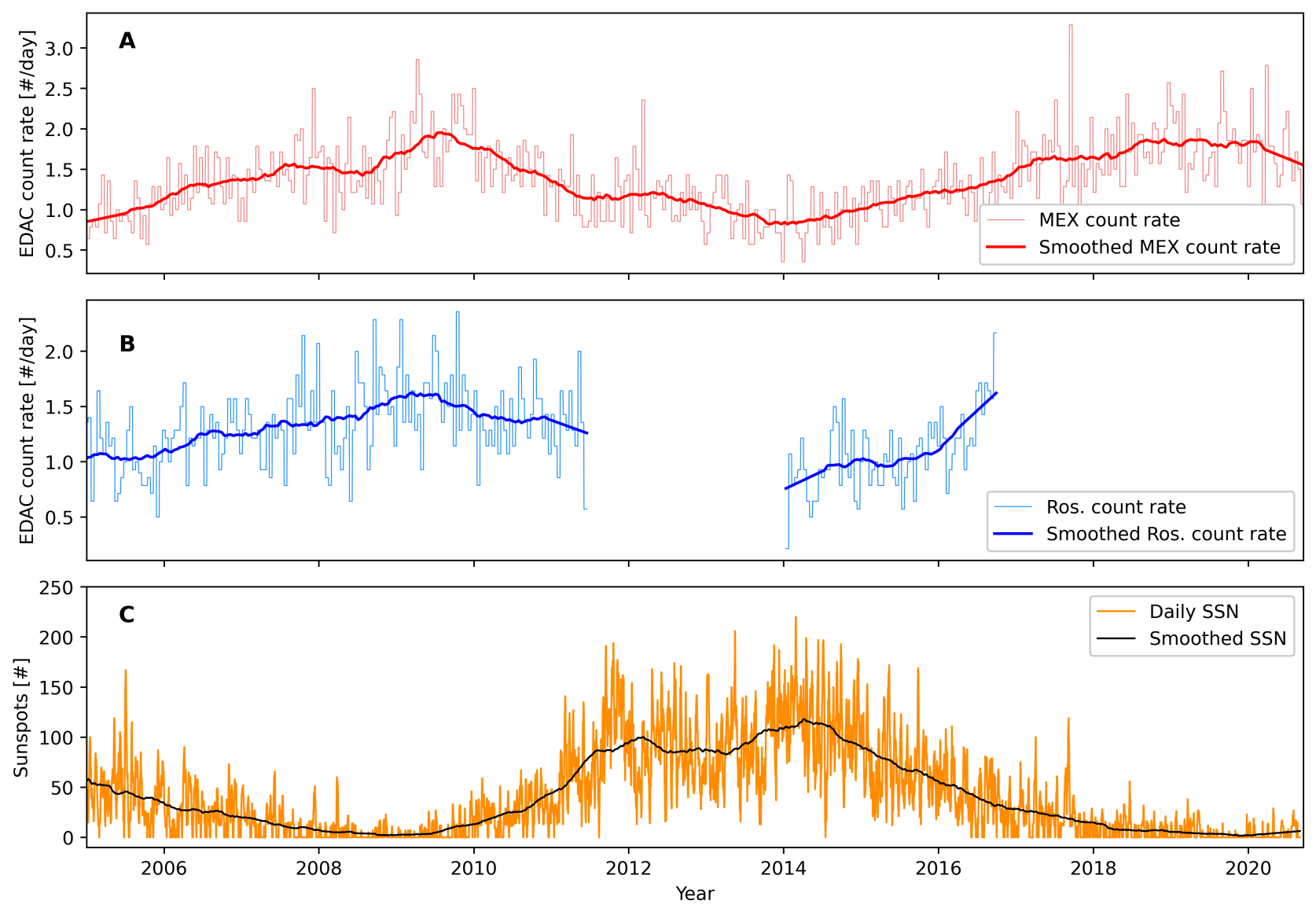

Fig. 3. Comparison between the two EDACs and the relevant solar cycle. Panel A: MEX EDAC daily count rates based on 14-day averages, with smoothed count rates. Panel B: Rosetta EDAC daily count rates based on 14-day averages, with smoothed count rates. Panel C: daily SSNs along with smoothed SSNs.

in Fig. 3C. From visual inspection, a pronounced anti-correlation can be discerned, which is further investigated in Fig. 4.

By inspection of Fig. 3, the GCR intensity minimum can be seen to occur during the time of highest solar activity at the very beginning of 2014, that is, during the polarity reversal phase of the solar magnetic dipole field (Janardhan et al. 2018). Due to the particularly strong warping of the heliospheric current sheet during solar maximum (Prölss 2004), cosmic ray access to the inner heliosphere is diminished during this time, causing the anti-correlation between GCR intensity and SSNs. The Rosetta and MEX EDACs exhibit very similar behaviours overall despite the difference in location. To investigate the EDAC-solar cycle relationship further, the MEX EDAC and SSN are compared in a correlation study in Fig. 4. The Rosetta EDAC is not used for this part of the analysis due to the long hibernation period interrupting the time series.

Figure 4 compares the MEX EDAC counter with solar activity over the course of 15 years. Panel A highlights the cyclic behaviour of the two time series, and the relationship is shown as a scatter plot in panel B with a clear linear relation. The daily EDAC count rate at Mars orbit during solar minimum is twice as high $\left(1.9\right.$ counts day $\left.{ }^{-1}\right)$ as the daily count rate during the months of maximum solar activity $\left(0.8\right.$ counts day $\left.{ }^{-1}\right)$. Panel $\mathrm{C}$ quantifies the lag, which is barely discernible by visual inspection in panel A, by cross-correlation analysis. A lag time of 5.5 months is obtained, which corresponds well with results from previous studies of solar modulation around the Earth; Bertucci et al. (2019) obtained a lag time of 6-8 months, and Ross \& Chaplin
(2019) found the lag to be $2-4$ months. The difference between the two might be due to the difference in energies they measured. Interestingly, the origin of the delayed GCR response remains unknown.

\subsection{GCR variations with radial distance}

The flux of cosmic rays increases when moving away from the Sun, but it is also modulated by the solar activity and the solar cycle. It will also differ depending on the measured energy interval. In this study we utilise EDACs on MEX and on Rosetta as it journeyed outwards in the Solar System. To obtain a GCR gradient independent of the solar cycle modulation, simultaneous EDAC measurements on multiple spacecraft at different locations are required. As the heliocentric distance of each spacecraft is known at all times, one can disentangle the radial effects from the solar cycle modulation effects observed with Rosetta by comparing the EDAC counters on each spacecraft at the same time. Heliocentric longitudinal and latitudinal variations in the GCR flux are considered to be negligible compared to the radial variation in this study (De Simone et al. 2011; Vos \& Potgieter 2016; Heber et al. 2009). To compare the MEX and Rosetta EDAC rates as a function of heliocentric distance, we calculated the difference in heliocentric distance between the spacecraft by subtracting the Mars-Sun distance from the Rosetta-Sun distance for each day of Rosetta's lifetime. The result is shown in Fig. 5, where the ratio between the Rosetta and MEX EDAC rates is plotted as a function of the difference between the solar distances 


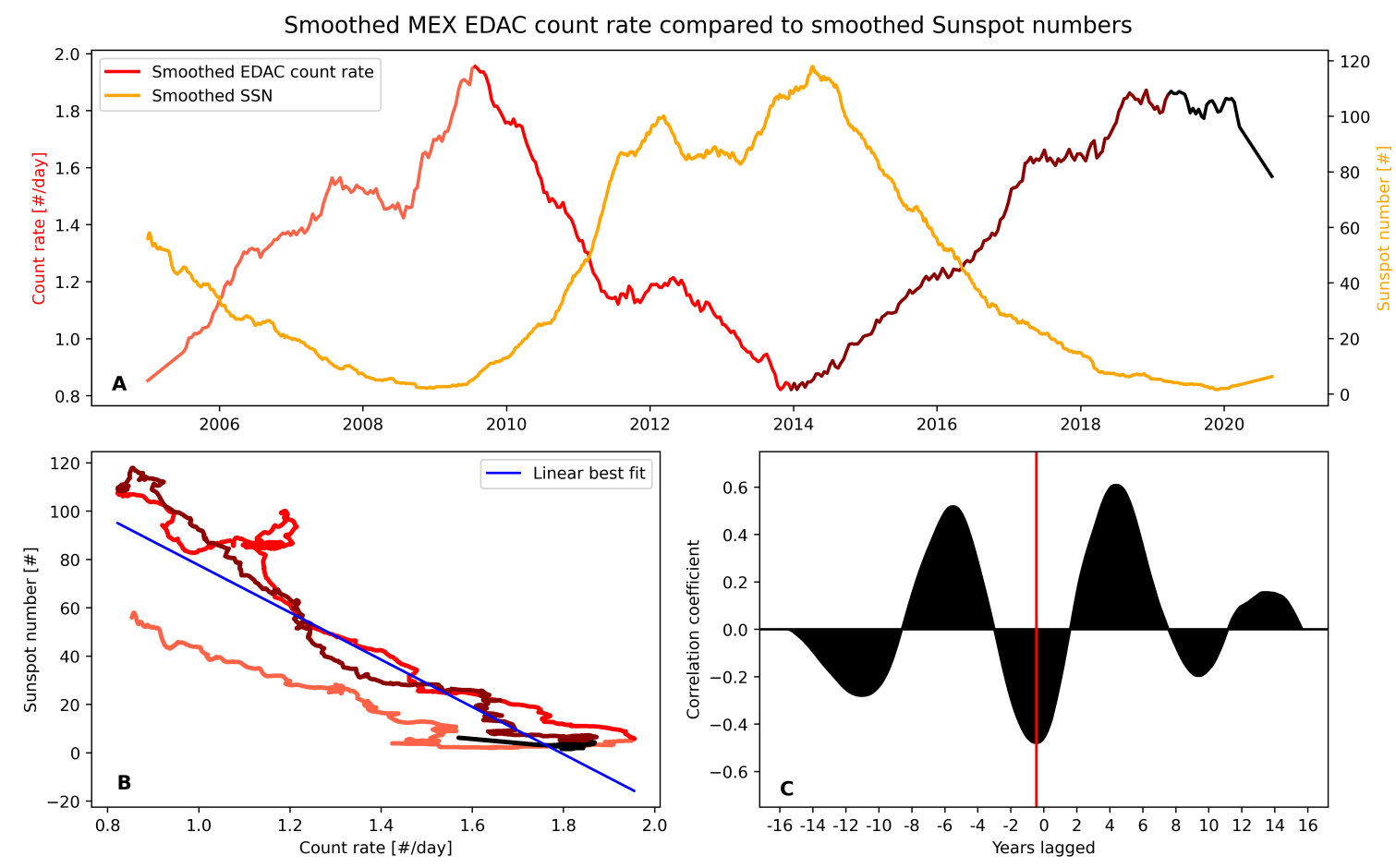

Fig. 4. Long-term solar cycle modulation of GCRs. Panel A: smoothed SSNs in orange along with smoothed MEX EDAC count rates in hues of red. The count rate is colour-coded according to the solar cycle phases to highlight the effect of solar modulation. The colours match the colours in panel B. Panel B: SSNs as a function of MEX count rates in hues of red as defined in panel A. The blue line is the linear best fit of the data points, indicating the relationship between the two parameters. Panel $C$ : cross-correlation between smoothed SSN and MEX count rates, illustrating a cyclic anti-correlated relationship, where the EDAC rate lags 5.5 months behind the solar cycle as measured by the SSN.

of Rosetta and Mars. The $x$-axis in Fig. 5 is such that $x=0$ indicates the heliocentric distance to the mean Martian orbit and any points at negative values translate to Rosetta being closer to the Sun than Mars.

A linear function was fitted to the data points where $x \geq$ 0 , with an obtained slope of $5.3 \%$, meaning the EDAC count rate, and subsequently the GCR flux, increased at an average rate of $5.3 \% \mathrm{AU}^{-1}$ for this dataset. If all measurements inside Mars orbit are also included, the rate becomes $3.9 \% \mathrm{AU}^{-1}$. In previous works (Honig et al. 2019; McDonald et al. 1997; Webber \& Lockwood 1991), it has been customary to take the natural logarithm of the count rate ratio when calculating a radial gradient of GCR fluxes. By doing this for the two cases - all measurements and only measurements at and beyond Mars slopes of $4.1 \%$ and $5.4 \%$ are obtained, respectively, which are similar to the values found in previous studies. The mean and median of these four slopes both round up to 4.7 , and thus our final estimate of the GCR radial gradient becomes $4.7 \pm 0.8 \%$.

As Rosetta's journey included a hibernation period, there are two additional end points in the time series, as indicated by the diamonds in Fig. 5. Immediately after the launch of Rosetta and after waking up at the end of hibernation, the relationship between the two EDAC rates differ from the remaining 14 years of data. This could be related to shortcomings of the smoothing algorithm at the edges or was possibly caused by cometary shielding for 2014, as discussed in Honig et al. (2019).

\section{Discussion}

In situ measurements of the radiation environment around Earth have been conducted for almost six decades, with Mariner 2 becoming the first satellite to travel from Earth to another planet (Neugebauer \& Snyder 1962). Since then, specialised radiation instrumentation has flown on multiple spacecraft, providing detailed data of the solar modulation of GCR activity at and around the near-Earth environment. Zeitlin et al. (2010) recorded, for the first time, solar modulation of GCR activity in Martian orbit from 2004 to 2007. Here we have confirmed, and expanded upon, those results. Several SPEs were also detected by Zeitlin et al. (2010), and complimenting those measurements (and others) with EDAC data will be the focus of future work.

Recently, the radiation dose rate was measured in Martian orbit with the FREND (Fine Resolution Epithermal Neutron Detector) instrument during the insertion phase of the ExoMars Trace Gas Orbiter. With this instrument, Semkova et al. (2018) observed changes in the GCR-related dose rate in accordance with the declining phase of the solar cycle. The GCR radiation dose has also been measured at the Martian surface by the MSLRAD instrument, where the solar cycle modulation was observed (Berger et al. 2020). The dose rate was found to be fairly similar to that on the Moon, although the presence of the Martian atmosphere shields the surface from lower-energy GCRs, resulting in a reduced modulation effect.

In this study we measure the solar cycle modulation of GCRs at Martian orbit and thus avoid the low-energy particle filtration effect of the Martian atmosphere. On the other hand, the spacecraft itself shields against the low-energy part of the GCR spectrum. Quantifying this effect is complicated and requires detailed knowledge of the spacecraft (geometry and components) and was not considered for this study.

As previously mentioned, longitudinal effects were assumed to be negligible (De Simone et al. 2011) for this work. In addition to longitudinal effects, Rosetta's trajectory does not at all times lie within the ecliptic plane $\left( \pm 8^{\circ}\right.$ latitude; Honig et al. 2019), 


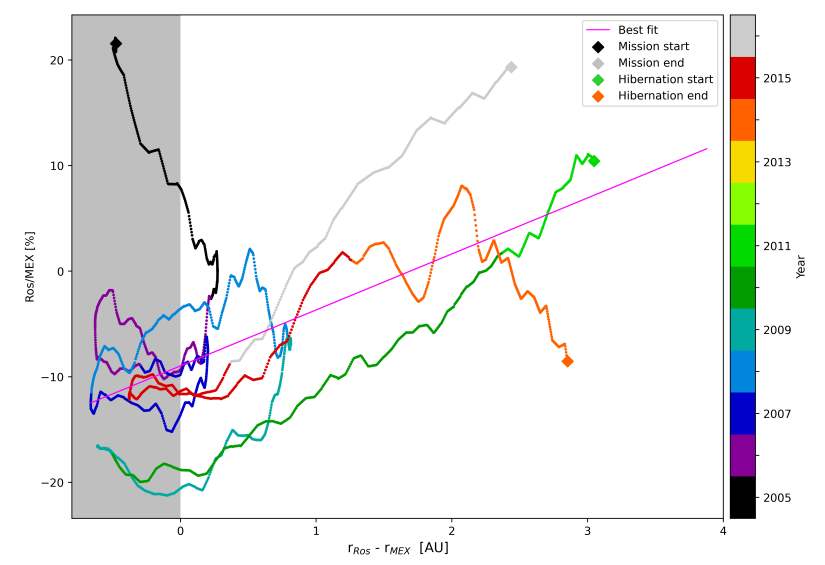

Fig. 5. Ratio of smoothed Rosetta and MEX EDAC count rates as a function of the difference in solar distance. The magenta line indicates the linear best fit of the data, with an obtained slope corresponding to a $5.3 \% \mathrm{AU}^{-1}$ increase in GCR intensity. Each colour indicates a full terrestrial year, except for 2016 (grey), which ends on September 29. The diamonds mark the start and end points of the Rosetta EDAC time series. The lightest shades of green and yellow are not present in the plot as they represent times when Rosetta was in hibernation. The grey shaded area for $x<0$ indicates when Rosetta was at or within mean Martian orbit. EDAC data points within this region were not included in the best-fit procedure illustrated in the figure.

and thus this adds to the already varying heliocentric latitude caused by the inclination of the Sun's rotational axis. MEX data was only used after orbit insertion around Mars, so radial and latitudinal effects are reduced to those caused by Mars' elliptical orbit and the inclination of the Sun's rotational axis. Latitudinal gradients, however, are known to be small (Heber et al. 2009). All heliocentric longitudes are covered. Thus, the effects these parameters might have on the EDAC counter are considered to be very small compared to solar cycle modulation and heliocentric distance effects.

Another aspect to consider is planetary shielding. Over the course of the MESSENGER mission, a neutron spectrometer was used for long-term study of GCR variations in the inner heliosphere (Lawrence et al. 2016). When the satellite was within a certain range of Mercury, the GCR intensity was observed to drop since the GCRs incident on the solid angle of the planet were intercepted and did not reach the spacecraft. Lawrence et al. (2016) therefore set a cautionary limit of $8000 \mathrm{~km}$ and abstained from using data when the spacecraft was within this range of the planet. MEX is in a highly elliptical quasi-polar orbit around Mars, with periapsis at $330 \mathrm{~km}$, apoapsis at $10500 \mathrm{~km}$, and a period of 7 hours. More than 5 of those 7 hours are spent at altitudes higher than $5000 \mathrm{~km}$, at which distance Mars subtends $4 \%$ of the total sky. Planetary shielding was therefore assumed to have a marginal effect on the MEX EDAC counter even when data from the full orbit were utilised and has therefore not been taken into account for either of the EDAC datasets considered here.

The solar cycle modulation of GCRs has been under intense study for decades (e.g., Forbush 1958; Usoskin et al. 1998; Bertucci et al. 2019), and though the existence of a time lag of GCR intensity relative to solar activity is well known, its origins remain elusive. In general, the lag is caused by the vast size of the heliosphere and the diffusive and drifting nature of the cosmic rays (Dorman \& Dorman 1967), though the details of the process are unexplained. The lag is larger for odd numbered solar cycles than for even numbered ones (Usoskin et al. 1998), which is related to the polarity of the 22-year magnetic cycle of the Sun. This study spans almost 1.5 cycles, including solar cycle 24 in its entirety. The polarity reversal normally occurs near solar maximum for each cycle, with one hemisphere flipping about 1 year before the other (Pishkalo 2019). The lag obtained here of 5.5 months during an even numbered cycle is smaller than the lag found by Bertucci et al. (2019) for the period 2008-2012, which follows conventional predictions, but slightly larger than what Ross \& Chaplin (2019) found for the same cycle (2-4 months).

The EDAC counter increase with heliocentric distance obtained by comparing the EDACs on MEX and Rosetta indicates an average GCR gradient of $4.7 \pm 0.8 \% \mathrm{AU}^{-1}$ between 2005 and 2020. The uncertainty stems from the calculation method used (simple count rate ratios or natural logarithm of said ratios) and whether or not data points Sunwards of the mean Mars orbit are included. This result confirms what dedicated radiation instrumentation on other spacecraft has previously measured. When Rosetta was at heliocentric distances equal to or smaller than mean Mars orbit, the spacecraft conducted several maneuvers and multiple fly-bys. Planetary shielding effects and the passing through Earth's Van Allen radiation belts on multiple occasions led us to differentiate between data points within and outside Martian orbit when Rosetta's trajectory was less convoluted. If all data points are included, the GCR gradient drops to $3.8 \% \mathrm{AU}^{-1}$.

By comparing three years of data from the Kiel Electron Telescope on the Ulysses spacecraft and the PAMELA (Payload for Antimatter Matter Exploration and Light-nuclei Astrophysics) instrument, De Simone et al. (2011) found a radial gradient of $2.7 \%$ per AU for particles in the range $1.6-1.8 \mathrm{GV}$ during the solar cycle minimum between cycles 23 and 24 . For the same time period and using the same instruments, Vos \& Potgieter (2016) found a radial gradient of $4.25 \% \mathrm{AU}^{-1}$. Gradients are generally stronger during the negative polarity phase when $A<0$ and the magnetic field has a radial inward component north of the current sheet, in this case during 20052014, and weaker for times with positive polarity. The recent work of Roussos et al. (2020) found similar values with Cassini data, with a peak gradient of $4 \% \mathrm{AU}^{-1}$ and an average gradient of $3.5 \% \mathrm{AU}^{-1}$ for $A<0$ in the period 2006-2014. Our measurements span multiple polarity periods, and the gradient found here should therefore be considered a long-term average. Moreover, the rigidity of those particles responsible for EDAC counts is currently not well understood. A quantitative comparison with other methods is thus still elusive. Nevertheless, we expect our results to be robust if we compare between EDAC counters on other spacecraft as long as these counters provide comparable amounts of shielding for the spacecraft memories.

Honig et al. (2019) found a GCR gradient anomaly during the comet-approach phase of the Rosetta mission, when the GCR count rate dropped by $8 \%$ and thus exhibited a negative relationship with heliocentric distance. Our results also indicate that the GCR flux was different before and after hibernation. This effect was tentatively attributed to cometary shielding by Honig et al. (2019), although the phenomenon requires further investigation. In 2005, Rosetta was still for the most part within Martian orbit, and the closest approach fly-by of Earth was also during this period, which might have caused the elevated EDAC rate compared to MEX at the same time (see Fig. 3).

In this analysis, EDAC counters from two spacecraft were utilised to infer the solar cycle modulation of GCRs in the heliosphere and the increase in GCR intensity with heliocentric distance. By including EDAC counters from additional spacecraft, the spatial and temporal coverage would increase drastically. The 
main caveat to using EDAC counters individually to infer GCR variations is the uncertain quantification of the energies or rigidities of the particles that are responsible for errors in the memory chips. The GCR spectrum covers a broad range of energies, and not all will be sufficient to penetrate the spacecraft and result in an EDAC increment. To understand this, knowledge of the 3D structure of the spacecraft is required. Since the solar effects on GCR variation is rigidity dependent, it is imperative to compare datasets with equal rigidities (De Simone et al. 2011). The particle energies that cause SEUs in one spacecraft might not be the same in another as it will depend on the location of the onboard computer and the shielding effect of the rest of the spacecraft. The spacecraft chosen for this study, Rosetta and MEX, are largely similar, designed with a large degree of commonality in several subsystems and with comparable solid-state mass memory units (Ferri et al. 2004), which gives us confidence in the results reported here. The potential scientific return from EDAC counters is irrefutable, and the benefits from supplementing the counters with science instruments are numerous.

\section{Conclusion}

Ground-based NMs on Earth have been utilised for decades to monitor the near-Earth GCR environment. Here we demonstrate that the EDAC engineering parameter can provide similar information from a multitude of locations in the Solar System. EDAC counters can be used to monitor the time evolution and 3D structure of the GCRs in the heliosphere.

We have shown that, as expected, the GCR variations at Mars follow a solar cycle modulation very similar to that observed at Earth, with twice as high daily count rates during solar minimum compared to solar maximum, for those energies that the EDAC counter is sensitive to. The GCR-induced EDAC signal was found to lag SSNs by approximately 5.5 months, comparable to previous results (Bertucci et al. 2019; Ross \& Chaplin 2019).

By comparing the Rosetta and MEX EDAC counters, we found that EDAC count rates increased by $4.7 \pm 0.8 \%$ per $\mathrm{AU}$, which is in good agreement with previous studies of Rosetta radiation monitor data (Honig et al. 2019). This behaviour is expected as the flux of GCRs increases with increasing heliocentric distance (e.g., Ferreira \& Potgieter 2004).

This work demonstrates the ability of EDAC counters and shows that they are well suited for GCR studies and can be used to identify long-term GCR variations in the heliosphere. Because all spacecraft are equipped with EDAC counters irrespective of their scientific objectives, EDAC data can provide information about the GCR flux at multiple locations in the heliosphere. In addition, shorter-term GCR variations in the form of Forbush decreases, as measured by EDACs, may inform on the propagation of large solar transient events (e.g., Sánchez-Cano et al. 2017; Witasse et al. 2017), particularly in those instances when there is no other instrumentation available to measure them Future work will need to quantify the effect of different shielding geometries on the EDAC count rate, and methods for cross-calibration between different spacecraft will be needed to improve the quality of EDAC data for scientific studies. Nevertheless, the opportunity offered by such multi-point measurements to improve the understanding of particle transport processes in the heliosphere should be followed up on.

Acknowledgements. E.W.K. thanks the internship program at ESA-ESTEC for the opportunity and financial support to carry out this project. E.W.K. has changed institutions while finishing this work, and is now at LATMOS/CNRS B.S.-C. and M.L. acknowledge support thought UK-STFC grant ST/N000749/1 B.S.-C. gratefully acknowledges ESA-ESTEC Faculty support. MEX EDAC data was provided by the ESOC Mars Express flight control team and is available upon request (Olivier.Witasse@esa.int). Rosetta data is available in the ESA planetary science archive. Neutron monitors of the Bartol Research Institute are supported by the National Science Foundation. We thank the Royal Observatory of Belgium and the World Data Center SILSO for providing freely accessible Sunspot number data.

\section{References}

Alania, M. V., Modzelewska, R., \& Wawrzynczak, A. 2014, J. Geophys. Res. Space Phys., 119, 4164

Berger, T., Matthiä, D., Burmeister, S., et al. 2020, J. Space Weather Space Clim., 10,34

Bertucci, B., Fiandrini, E., Khiali, B., \& Tomassetti, N. 2019, in Proceedings of Science, Madison, WI, USA, PoS(ICRC2019)1162

Burger, R. A., Kruger, T. P. J., Hitge, M., \& Engelbrecht, N. E. 2008, ApJ, 674, 511

Center, S. W. D. 2020, International Sunspot Number Monthly Bulletin and online catalogue

Chicarro, A., Martin, P., \& Trautner, R. 2004, The Mars Express Mission: an Overview, 3

De Simone, N., Di Felice, V., Gieseler, J., et al. 2011, Astrophys. Space Sci. Trans., 7, 425

Dorman, I. V., \& Dorman, L. I. 1967, J. Geophys. Res., 72, 1513

Durante, M., \& Cucinotta, F. A. 2011, Rev. Mod. Phys., 83, 1245

Engel, J., Gaisser, T. K., Lipari, P., \& Stanev, T. 1992, Phys. Rev. D, 46, 5013

Ferreira, S. E. S., \& Potgieter, M. S. 2004, ApJ, 603, 744

Ferri, P., Denis, M., Accomazzo, A., \& Warhaut, M. 2004, Space OPS 2004 Conference (Montreal, Quebec, Canada: American Institute of Aeronautics and Astronautics)

Feynman, J., \& Gabriel, S. B. 2000, J. Geophys. Res.: Space Phys., 105, 10543

Forbush, S. 1958, J. Geophys. Res., 63, 651

Gazis, P. R. 1996, Rev. Geophys., 34, 379

Glassmeier, K.-H., Boehnhardt, H., Koschny, D., Kührt, E., \& Richter, I. 2007, Space Sci. Rev., 128, 1

Hassler, D. M., Zeitlin, C., Wimmer-Schweingruber, R. F., et al. 2012, Space Sci. Rev., 170, 503

Hassler, D. M., Zeitlin, C., Wimmer-Schweingruber, R. F., et al. 2014, Science, 343, 1244797

Heber, B., Kopp, A., Gieseler, J., et al. 2009, ApJ, 699, 1956

Honig, T., Witasse, O., Evans, H., et al. 2019, Ann. Geophys., 37, 903

Janardhan, P., Fujiki, K., Ingale, M., Bisoi, S. K., \& Rout, D. 2018, A\&A, 618, A148

Jiggens, P., Clavie, C., Evans, H., et al. 2019, Space Weather, 17, 99

Lawrence, D. J., Peplowski, P. N., Feldman, W. C., Schwadron, N. A., \& Spence, H. E. 2016, J. Geophys. Res.: Space Phys., 121, 7398

Limes, G., Christa, S., \& Pires, C. 2015, EDAC Events During the LADEE Mission (IEEE), 1

McDonald, F. B., Ferrando, P., Heber, B., et al. 1997, J. Geophys. Res.: Space Phys., 102, 4643

Modzelewska, R., Iskra, K., Wozniak, W., Siluszyk, M., \& Alania, M. V. 2019, Sol. Phys., 294, 148

Neugebauer, M., \& Snyder, C. W. 1962, Science, 138, 1095

Pishkalo, M. I. 2019, Sol. Phys., 294, 137

Potgieter, M. 2013, Liv. Rev. Sol. Phys., 10, 3

Prölss, G. W. 2004, Physics of the Earth's Space Environment (Berlin, Heidelberg: Springer, Berlin Heidelberg)

Richardson, J. D., Wang, C., \& Paularena, K. I. 2001, Adv. Space Res., 27, 471 Ross, E., \& Chaplin, W. J. 2019, Sol. Phys., 294, 8

Roussos, E., Dialynas, K., Krupp, N., et al. 2020, ApJ, 904, 165

Sánchez-Cano, B., Hall, B. E. S., Lester, M., et al. 2017, J. Geophys. Res.: Space Phys., 122, 6611

Savitzky, A., \& Golay, M. 1964, J. Anal. Chem., 36, 1627

Schwadron, N. A., Blake, J. B., Case, A. W., et al. 2014, Space Weather, 12, 622

Semkova, J., Koleva, R., Benghin, V., et al. 2018, Icarus, 303, 53

Shirvani, P. P., Saxena, N. R., \& McCluskey, E. J. 2000, IEEE Trans. Reliab., 49, 273

Simpson, J. A. 2000, Space Sci. Rev., 93, 11

Usoskin, I. G., Kananen, H., Mursula, K., Tanskanen, P., \& Kovaltsov, G. A. 1998, J. Geophys. Res.: Space Phys., 103, 9567

Usoskin, I. G., Gil, A., Kovaltsov, G. A., Mishev, A. L., \& Mikhailov, V. V. 2017, J. Geophys. Res.: Space Phys., 122, 3875

Vos, E. E., \& Potgieter, M. S. 2016, Sol. Phys., 291, 2181

Webber, W., \& Lockwood, J. 1991, J. Geophys. Res., 96, 15,899

Wibberenz, G. 2002, J. Geophys. Res., 107, 1353

Witasse, O., Sánchez-Cano, B., Mays, M. L., et al. 2017, J. Geophys. Res.: Space Phys., 122, 7865

Zeitlin, C., Boynton, W., Mitrofanov, I., et al. 2010, Space Weather, 8, S00E06 